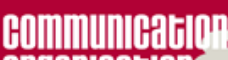

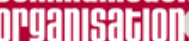

Communication et organisation

Revue scientifique francophone en Communication organisationnelle

$52 \mid 2017$

Communauté, sociabilité et bien commun: approche internationale

\title{
Nicolas Oliveri, Apprendre en ligne, quel avenir pour le phénomène MOOC?
}

Paris, Éditions l'Harmattan, Collection « Communication et civilisation », $126 \mathrm{p}$.

\section{Anne Lubnau-Wimez}

\section{OpenEdition}

\section{Journals}

Édition électronique

URL : https://journals.openedition.org/communicationorganisation/5789

DOI : 10.4000/communicationorganisation.5789

ISBN : 979-10-300-0257-7

ISSN : $1775-3546$

\section{Éditeur}

Presses universitaires de Bordeaux

\section{Édition imprimée}

Date de publication : 1 décembre 2017

Pagination : 241-246

ISBN : 979-10-300-0163-1

ISSN : $1168-5549$

Référence électronique

Anne Lubnau-Wimez, " Nicolas Oliveri, Apprendre en ligne, quel avenir pour le phénomène MOOC ? ", Communication et organisation [En ligne], 52 | 2017, mis en ligne le 01 décembre 2017, consulté le 09 décembre 2021. URL : http://journals.openedition.org/communicationorganisation/5789 ; DOI : https://doi.org/10.4000/communicationorganisation.5789 
vocation illustrative, ces retours d'expérience recèlent une dimension critique. Par exemple, l'un des experts s'oppose à une vision béate et simpliste du numérique. Il souligne qu'en raison de sa viralité, de sa rapidité de diffusion et du caractère désinhibant de l'expression en ligne, le web constitue autant une ressource qu'une menace pour la communication organisationnelle.

En conclusion, ce livre très complet cible principalement les professionnels -spécialistes de la communication ou issus d'autres fonctions et désirant acquérir des connaissances dans ce domaine-et les étudiants. Loin d'accréditer une vision instrumentale réduisant la communication à ses outils, l'auteur consacre une large part de l'ouvrage à la réflexion stratégique et à la démarche d'audit, preuve de sa lucidité face aux effets de mode de la profession. Le propos est exhaustif, bien documenté et soulève en creux plusieurs questions : celles des paradoxes engendrés par une communication ciblant plusieurs publics simultanément (salariés, financeurs et parties prenantes), des inconvénients et régulations posés par le développement de la communication numérique etc. Enfin, l'utilisation d'un langage clair permet une lecture fluide et rapide.

\section{Delphine DUPRÉ Doctorante contractuelle en SIC, Laboratoire MICA $\in A 4426$ Université Bordeaux Montaigne}

\section{Nicolas OLIVERI, Apprendre en ligne, quel avenir pour le phénomène MOOC? Éditions l'Harmattan, collection «communication et civilisation »126p., 14,50 €. ISBN : 978-2-343-08640-8.}

Dans sa préface, Paul Rasse souligne que cet ouvrage donne une idée précise du territoire qu'occupent les $\mathrm{MOOC}^{1}$ et plus largement l'enseignement à distance en France. Il s'interroge sur leur avenir: les MOOC vont-ils continuellement se réinventer pour au final se pérenniser sous une forme spécifique avec des contenus donnés?

Nicolas Oliveri dresse l'historique de l'informatique au sein du système éducatif, et des querelles de marché entre entreprise française et la très emblématique société APPLE. Le début de lère informatique dans le milieu scolaire date des années 80. « Nous pouvons constater que l'avancée technologique de l'apprentissage, qui se faisait essentiellement avec une craie et une ardoise, est aujourd'hui passée à l'usage du tableau blanc interactif ». Mais cela garantit-il l'avancée d'un apprentissage de qualité ?

Nicolas Oliveri présente les enjeux analyse les freins et des leviers qui accompagnent le développement des MOOC, au niveau des acteurs:

1 Cours en ligne ouvert et massif. 
apprenants, enseignants, entreprises MOOC, ainsi qu'au niveau de leur reconnaissance académique.

Plusieurs facteurs participeraient au développement des MOOC du côté de l'apprenant: le degré de motivation d'un apprenant, le niveau de compétences de base du logiciel d'apprentissage mais aussi le niveau de formation de l'encadrant. À cela, il faut rajouter le degré d'équipement des apprenants en matériel informatique et le développement du réseau internet, selon le territoire géographique qui participent au succès des MOOC.

Du côté des enseignants, leur réticence proviendrait de plusieurs facteurs : renoncer à la pédagogie du tout face à face, les avancées constantes techniques des équipements et des logiciels qui dépassent les compétences de leurs utilisateurs. Un enseignant en présentiel coûte moins cher. Généraliser l'apprentissage par le numérique à distance coûte cher, mais devient intéressant, dès lors que le nombre d'apprenants est plus nombreux à s'inscrire à l'enseignement à distance.

Il existe deux géants internationaux: Udacity et Coursera. Nicolas Oliveri décrit ce qui fait leur hégémonie tant au niveau du niveau des inscrits, que du contenu des MOOC (sciences formelles: intelligence artificielle, programmation informatique, physique..)

Les MOOC, dans le monde anglo-saxon, se développent davantage qu'en France, car l'expérience et les compétences de l'étudiant, sa polyvalence sont privilégiées sur le marché du travail.

En France, la culture des vertus du diplôme reste bien ancrée. Cependant, même si le développement des MOOC est moins spectaculaire en France, il s'appuie sur des bases institutionnelles solides. Devant l'engouement et le nombre d'inscrits grandissants, une plateforme d'un groupement d'intérêt public appelée «Fun MOOC » a vu le jour à l'été 2015.

Depuis 2015, 53 MOOC français et francophones développés par les Universités et les grandes écoles ont vu le jour (MOOC en philosophie, géographie, histoire, santé, environnement, droit management et entreprenariat, et pédagogie du numérique).

Nicolas Oliveri présente dans cette partie, les quatre dispositifs de MOOC existants mais aussi les quatre modèles de bussiness models sur lesquels ils reposent en décrivant leurs limites:

- $5 \%$ des apprenants inscrits à un MOOC le fréquentent jusqu'à son terme. Un tiers de français ignore ce qu'est un MOOC.

- les small private online classes (SPOC), petits cours privés privilégiés en ligne répondraient à un double objectif : augmenter le taux de complétion, et être rentable pour le propriétaire de la plate-forme. Cependant, il reste à définir un format commercial et éditorial reconnu tenant compte des contraintes juridiques de légitimité. 
- les COOC (corporate online open courses) seront à destination des professionnels et des grands groupes dans un format propre à asseoir leur réseau.

- Les MIMO ou mini MOOC auront une durée maximale de 20 minutes pour éviter les abandons.

De nombreux directeurs de ressources humaines dans les entreprises françaises n'ont pas intégré le concept de MOOC, ni pour les besoins en formation, ni dans les compétences à cibler chez les candidats à recruter. Les instances nationales de formation continue ne reconnaissent pas les MOOC.

Dans l'enseignement supérieur, le taux de réussite à un MOOC est faible, ce qui nécessiterait davantage d'encadrement. La faible interactivité entre professeurs et apprenants semble limiter à quelques commentaires, liée en partie par la barrière de la langue.

Les critères d'évaluation sont aussi discutables, car bien souvent, cela résume à indiquer le nombre d'erreurs, et non le pourquoi. Une méthode d'évaluation basée sur le raisonnement clinique propose à l'apprenant plusieurs solutions à hiérarchiser.

L'auteur présente ensuite une série d'entretiens menés auprès de spécialistes du secteur éducatif usagers des MOOC.

\section{Mathieu Cisel doctorant à l'ENS de Cachan prépare une thèse sur les MOOC.}

Il constate que la France est en retard par rapport à ses voisins, le monopole est détenu par le Centre national d'enseignement à distance. En Angleterre, c'est la télévision qui assurait les cours à distance et c'est le même producteur qui est devenu leader des MOOC.

À part Fun et Coursera, l'impact des MOOC en France est nul. Il cite un module " gestion de projet " qui fait exception et intègre grand nombre de formations.

Il plaide en faveur d'une politique nationale volontariste qui mette en accord tous les acteurs : universités- grandes écoles- ministère- entreprisesformation continue.

En ce qui concerne l'évaluation, les MOOC sont davantage un moyen de formation et d'entrainement que de certification de compétences. Le système de notation réalisé de façon automatique a ses limites. Une double notation s'avère la plus objective.

\section{Daniel Moati, enseignant - chercheur HDR, Université de Nice Sophia- Antipolis.}

Ancien enseignant documentaliste en collège et en lycée, il revient sur l'historique de l'introduction des NTIC et des cours en ligne. En ce qui concerne les MOOC, il fait part de sa longue expérience de ce qui marche et de ce qui ne marche pas dans le secondaire et à l'université, en s'appuyant sur les résultats obtenus de la part des apprenants, y compris à l'international. 
Lutilisation des fonctionnalités des appareils numériques est limitée par les digitales natives, car ils les utilisent presque exclusivement dans un cadre ludique. Les élèves n'en connaissent pas toujours les dangers. Les élèves français perdent des places chaque année dans le rapport PISA (programme for international student assessment) mené par l'OCDE vérifiant l'acquisition des compétences et de savoirs des jeunes de 15 ans. Les banlieues dites sensibles font entrainer à la baisse les résultats. Pour les élèves en difficultés, les actions proactives de l'adulte ont plus d'effets que l'enseignement numérique à lui seul. L'identification de l'élève à l'enseignant et au groupe d'élève compte, car ce sont des hommes et des femmes en devenir.

Aux États-Unis et en Angleterre, les enquêtes sur les résultats des MOOC sont négatives. Un budget de 500 millions de dollars aux USA avec APPLE a été annulé en 2014, information discrètement relayée par le café pédagogique, association de promotion du numérique éducatif en France.

\section{Catherine Mongenet, chargée de mission FUN, France Université numérique.}

La mission du ministère en 2013 était de créer une plateforme numérique. Au départ, il s'agissait d'une plateforme open source Edx, où les contributeurs universitaires pouvaient contribuer et déposer leur MOOC. Devant l'engouement et le nombre d'inscrits grandissants, une plateforme d'un groupement d'intérêt public appelée « Fun MOOC » a vu le jour à l'été 2015.

Les MOOC selon Catherine Mongenet sont à la fois du e-learning, qui s'enrichit grâce aux activités sur les forums, sur les wikis et ce grâce au web 2.0. L'enseignant, accomplit un effort pédagogique conséquent et de grande qualité. C'est aussi le résultat d'un travail collectif d'une équipe autour de ce professeur, qui rassemble des ingénieurs pédagogiques, spécialistes de l'audiovisuel, style " community management ».

Elle fait état de deux enquêtes ont eu lieu en France, respectivement en mai 2014 et en mai 2015. L'engouement pour les utilisateurs de MOOC est évident : les apprenants auront l'occasion de mettre en pratique, ce qu'ils ont appris et sont prêts à s'inscrire à un nouveau MOOC. Parmi eux, il y a une majorité d'actifs (60\%) et $12 \%$ d'étudiants.

Sur la plateforme FUN, des dispositifs de certification valident le travail fourni et les compétences acquises ont été déployées début 2016. Il est possible de valider deux crédits European credits transfer system «ECTS », dans le cadre de leur cursus universitaire.

Le monde de l'entreprise s'y implique. Trois exemples concrets :

- les recruteurs qui scrutent sur le curriculum vitae des étudiants les MOOC suivis.

- l'organisme paritaire collecteur agréé des industries agroalimentaires qui collecte la taxe sur la formation continue de ce secteur et propose des plans de formation aux salariés avec MOOC. 
- l'Agence de l'environnement et de la maîtrise de l'environnement et de l'énergie "ADEME », va mettre en place un MOOC dédié au " bâtiment durable ", qui concernera un ensemble de métiers : le chauffagiste, le plombier zingueur, le couvreur, l'architecte...

\section{En conclusion}

Lenjeu de la pérennité des MOOC est multiple : leur identité repose à la fois sur un format commercial et éditorial, qui allie spécificités culturelles, pédagogiques, managériales et marketing de l'établissement (système éducatif, universités, grandes écoles) qui est à l'origine de cette plate-forme d'apprentissage à distance destinée à des étudiants salariés ou éloignés géographiquement. Cependant, c'est aussi un moyen de démocratisation de l'éducation en facilitant l'accès au savoir au plus grand nombre.

Une dernière contrainte est de veiller à un strict respect des données personnelles des apprenants en ligne.

Les étudiants de la francophonie sont chaque année plus nombreux à s'y inscrire. D’une manière générale, les MOOC bénéficieront au cours et TP en présentiel, car ils auront été suivis et compris au préalable. Du coup, les projets, les études de cas, et les TP construits par les étudiants seront plus qualitatifs.

$\mathrm{Au}$ total, l'écosystème de l'entreprise est en train de s'emparer des MOOC pour la formation continue et le recrutement de ses salariés.

Anne LUBNAU WIMEZ

Docteure en sciences de l'information et de la communication et chercheures associée Université Bordeoux Montaigne Laboratoire de recherche Médiation Information Communication Art (MICA) Enseignante titulaire en sciences et techniques médicosociales 